\title{
Theoretical substantiation of the explosive crushing of particles of tungsten-cobalt alloys
}

\author{
Volodymyr Kurinnyi ${ }^{1}$, Ihor Harkusha ${ }^{1}$, and Serhii Musiienko ${ }^{2, *}$ \\ ${ }^{1}$ Dnipro University of Technology, 49005, Dnipro, Dmytra Yavornytskoho Ave., 19, Ukraine \\ ${ }^{2}$ Institute of Geotechnical Mechanics named by N. Poljakov of National Academy of Sciences of \\ Ukraine, 49005, Dnipro, Simferopolska Str., 2a, Ukraine
}

\begin{abstract}
The mechanisms of the processes occurring during the explosive crushing and softening of pieces of solid tungsten-cobalt alloys are considered. The parameters of the stress field required for their effective destruction are recorded. The estimation of the duration of the process of destruction in a given section of the explosive charge is carried out. A method is proposed for calculating the A and $\mathrm{m}$ coefficients in the Theta impact shock compressibility equation for tungsten-cobalt alloys and their value for the TC8 alloy is obtained. The parameters of strong compression waves in the TC8 alloy and steel at pressures in detonation products of 5 and $1 \mathrm{GPa}$ are estimated in cases of perpendicular and tangential wave incidence on the surface of the piece. The analysis of the processes occurring in a steel pipe filled with explosives and pieces of TC8 alloy was carried out. The work obtained the results needed by experimenters and explosives practitioners for developing designs of crushing charges for pieces of tungsten-cobalt alloys.
\end{abstract}

\section{Introduction}

In Ukraine cobalt and tungsten are not mined. Taking into account their high cost, it is advantageous to process scrap and lump waste of tungsten-cobalt (TC) alloys. Since tungsten carbide has high hardness and abrasiveness, crushing and grinding scrap pieces requires a large amount of energy. At the same time, equipment is significantly worn out.

Explosive destruction allows not only crushing pieces of TC scrap, but significantly weakening them. It is known that with the explosive destruction of pieces of TC alloys the grinding time decreases at least 20 times.

The microstructure of tungsten-cobalt alloys (WC-Co) is two-phase. The alloy consists of crystals of high-hard tungsten carbide (WC) and a cementing relatively soft phase of metallic cobalt (Co). Therefore, during the regeneration of tungsten and cobalt from secondary raw materials by an explosion, the main energy consumption falls on the destruction of WC grains. Grain size WC 1-3 microns.

Pieces of hard TC alloy are non-uniform in local strength. Under quasistatic loading, single cracks occur, connecting the least durable elements of the piece. As the loading rate increases, an increasing number of cracks develop. There is a destruction and softening of

\footnotetext{
${ }^{*}$ Corresponding author: mspdop@i.ua
} 
the sample. The minimum size of particles into which a piece breaks down is proportional to the rate of increase of stress and their size. Under explosive loading, the stress gradient modulus can reach a value of $10^{14} \mathrm{~Pa} / \mathrm{m}$.

\section{Methods}

In solving this problem, the methods of explosion physics, continuum mechanics, rock mechanics, gas and thermodynamics were used.

\section{Results and discussion}

In a compression wave in a substance on the surface separated from the wave front at a distance $r_{s}$, shear deformations occur and the substance is cut off almost instantly. The minimum particles size $r_{m}$, into which a piece of hard TC alloy is destroyed can be estimated by the formula:

$$
r_{m} \approx \sigma_{s} /(\partial p / \partial r)
$$

where $\sigma_{\mathrm{s}}$-the ultimate strength of the alloy during the shear; $p$ - the pressure in the compression wave; $r$ - the distance.

By a formula similar to (1), one can estimate the particle size in the unloading wave (extension), i.e. observe breakaway. In spherical and cylindrical compression waves, cracks grow, perpendicular to the wave front, the distance between which is proportional to the projections of the stress gradient in the same direction. For effective crushing and softening of pieces of hard TC alloy, you can write the parameters of the stress field in the form:

$$
\begin{gathered}
1 \mathrm{GPa} \leq p \leq 10 \mathrm{GPa} ; 10^{10} \mathrm{~Pa} / \mathrm{m} \leq d p / d r \leq 10^{12} \mathrm{~Pa} / \mathrm{m} ; \\
10^{10} \mathrm{~Pa} / \mathrm{s} \leq d \sigma / d t \leq 10^{15} \mathrm{~Pa} / \mathrm{s}, t \approx 20 \mu \mathrm{s} .
\end{gathered}
$$

At the same time, it was assumed that the particle diameter $d$, on which a piece of TC alloy is destroyed, should be within $1 \mu \mathrm{m}<d<5 \mathrm{~mm}$, and the particles are not weakened. It should be noted that with explosive crushing and subsequent grinding, about $10 \%$ of the alloy particles have high strength. They do not contain pores and microcracks.

Consider the processes occurring in the explosive crushing of TC8, the most popular WC-Co alloy containing $92 \%$ of tungsten carbide and $8 \%$ of cobalt.

The alloy density is $\rho_{0}=14800 \mathrm{~kg} / \mathrm{m}^{3}$, and the velocities of the stress waves in tungsten carbide and cobalt, respectively, are $C_{1}=4250 \mathrm{~m} / \mathrm{s}, C_{2}=4630 \mathrm{~m} / \mathrm{s}$.

The speed $C_{0}$ of the longitudinal wave in the TC8 alloy can be found from the formula obtained by the authors:

$$
C_{0}=\left(\rho_{0}\left(\frac{1-\alpha}{\rho_{1} C_{1}^{2}}+\frac{\alpha}{\rho_{2} C_{2}^{2}}\right)\right)^{-\frac{1}{2}}=4200 \mathrm{~m} / \mathrm{s},
$$

where $\rho_{1}, \rho_{2}$ are the densities of tungsten carbide and cobalt, respectively; $\alpha=0.08$ is the mass fraction of cobalt in the alloy.

To calculate the parameters of the waves is convenient to use the equation of shock compressibility in the form of Theta:

$$
p=A\left[\left(\frac{\rho}{\rho_{0}}\right)^{m}-1\right]
$$


where $p$ - pressure; $A, m$ - coefficients; $\rho_{0}, \rho$ are, respectively, the density of the TC alloy at atmospheric pressure and pressure $p$. The coefficients $A, m$ can be determined using the experimental dependence of the density $\rho$ on pressure for tungsten carbide and cobalt [1], the formula for the velocity of stress waves:

$$
C=\sqrt{\frac{m A}{\rho}}
$$

and equations (4) for hard alloy:

$$
\left(\frac{p_{m}}{\rho_{0} C^{2}}+1\right)^{-1 / m}=(1-\alpha)\left(\frac{p}{A_{1}}+1\right)^{-1 / m_{1}}+\alpha\left(\frac{p}{A_{2}}+1\right)^{-1 / m_{2}}
$$

where indices $0,1,2$ denote values for alloy, tungsten carbide and cobalt, respectively.

From the tables (see [1]) and formula (4) it was obtained that $m_{1}=4.04$ and $m_{2}=4.2$. From formula (5) it follows that $A_{0}=6.46 \cdot 10^{10} \mathrm{~Pa}$.

It was shown that at the pressures under consideration $(p<10 \mathrm{GPa})$, a shock wave (SW) does not arise in TC alloys. A strong compression wave (SCW) arises, which differs from the shock wave in that the parameters - pressure, density, mass velocity, entropy, etc. - do not experience a jump at the wave front. The values of all the SCW parameters decrease from the wave nucleation surface to its front. If we use the formula Theta of the shock compressibility of a substance, then at pressures far exceeding the dynamic yield strength of the substance, the equations for compression and rarefaction waves in gases or compressible liquids can be used. In this case, the initial parameters of the SCW and SW coincide.

Let us determine the initial parameters of the SCW in the TC8 alloy with a perpendicular incidence of the detonation wave (DW) on its surface from the equations for the SW [1]:

$$
\begin{gathered}
u_{s}=\frac{D}{n+1}\left(1-\sqrt{2 n} \frac{\left(p_{s} / p_{\alpha}\right)-1}{\sqrt{(n+1)\left(p / p_{\alpha}\right)+(n-1)}}\right)=\sqrt{\frac{p_{s}}{p_{0}}\left[1-\left(1+\left(\frac{p_{s}}{A_{0}}\right)^{-1 / m_{0}}\right)\right]}, \\
p_{\mathrm{s}}=\rho_{0} u_{\mathrm{s}} D_{\mathrm{s}} .
\end{gathered}
$$

Here $D$ is the explosive detonation velocity; $u_{\mathrm{s}}$ is the mass velocity of the TC alloy and detonation products (DP); $n$ is the adiabatic index of DP; $p_{\alpha}-$ pressure DP in the front; $\rho_{0}-$ TC8 alloy density; $m_{0}, A_{0}$ are the coefficients in the Theta equation; $p_{\mathrm{s}}$ is the pressure in the front of the shock wave; $D_{\mathrm{s}}$ is the speed of the shock wave.

As a result of a numerical solution for the case $D=5000 \mathrm{~m} / \mathrm{s}, p_{\alpha}=5 \mathrm{GPa}, n=3$, it was obtained that $u_{\mathrm{s}}=171.7 \mathrm{~m} / \mathrm{s}, p_{\mathrm{s}}=10.73 \mathrm{GPa}, D_{\mathrm{s}}=4220 \mathrm{~m} / \mathrm{s}$. If we consider that SCW occurs in the alloy, the equations for the initial parameters take the form:

$$
\begin{aligned}
& u_{s}=\frac{2}{m_{0}-1}\left(C\left(p_{s}\right)-C_{0}\right)=\frac{2 C_{0}}{m_{0}-1}\left(\left(1+\frac{p_{s}}{A_{0}}\right)^{\frac{m-1}{2 m}}-1\right)= \\
& \frac{D}{n+1}\left(1-\sqrt{2 n} \frac{p_{s} / p_{\alpha}-1}{\sqrt{(n+1)\left(p / p_{s}\right)+(n-1)}}\right), C\left(p_{s}\right)=C_{0}\left(1+\frac{p_{s}}{A_{0}}\right)^{\frac{m-1}{2 m}} .
\end{aligned}
$$


Here, $C_{0}, C\left(p_{\mathrm{s}}\right)$ is, respectively, the velocity of the elastic wave in the TC8 alloy and the velocity of the compression wave at pressure $p_{\mathrm{s}}$.

Solution of equations (8) gives $u_{\mathrm{s}}=171.7 \mathrm{~m} / \mathrm{s}, p_{\mathrm{s}}=10.73 \mathrm{GPa}, C\left(p_{\mathrm{s}}\right)=4257 \mathrm{~m} / \mathrm{s}$.

The difference in the $D_{\mathrm{s}}$ and $C\left(p_{\mathrm{s}}\right)$ values is explained by the fact that $D_{\mathrm{s}}$ does not change, and $C\left(p_{\mathrm{s}}\right)$ decreases almost to $C_{0}$ in the front of the SCW. At a pressure of $p=$ $1 \mathrm{GPa}$, the SCW parameters are equal: $p_{s}=2.34 \mathrm{GPa}, u_{s}=31.37 \mathrm{~m} / \mathrm{s}, C=4058.5 \mathrm{~m} / \mathrm{s}$.

In the case when the DW moves parallel to the surface of the TC8 piece, the SCW parameters are found from the equations [1]:

$$
u=\frac{2 n D}{n^{2}-1}\left(1-\left(\frac{p}{p_{\alpha}}\right)^{\frac{n-1}{2 n}}\right)=\sqrt{\frac{p}{p_{0}}\left(1-\left(1+\frac{p}{A_{0}}\right)^{-1 / m_{0}}\right)}
$$

The solution of equations (9) gives $u=77 \mathrm{~m} / \mathrm{s}, p=4.66 \mathrm{GPa}, C(p)=4115 \mathrm{~m} / \mathrm{s}$.

When $p=1 \mathrm{GPa}$, the parameter values are equal:

$$
u_{1}=16.5 \mathrm{~m} / \mathrm{s}, p_{1}=9.85 \cdot 10^{8} \mathrm{~Pa}, C_{1}=4028 \mathrm{~m} / \mathrm{s} .
$$

Usually, pieces of TC8 are placed in a steel pipe, so it is necessary to know the parameters of SCW in steel. According to formulas (8), it is possible to determine the parameters of the SCW in the case of a perpendicular drop of the DW on a steel surface $\left(A=2.1110^{10} \mathrm{~Pa}, m=5.5, C_{0}=5000 \mathrm{~m} / \mathrm{s}, \rho=7840 \mathrm{~kg} / \mathrm{m}^{3}\right)$.

In this case, $u_{s}=293 \mathrm{~m} / \mathrm{s}, p_{s}=9.93 \mathrm{GPa}, C=5937 \mathrm{~m} / \mathrm{s}$ with $p_{\alpha}=5 \mathrm{GPa}$.

If we assume that $p=1 \mathrm{GPa}$, then:

$$
u_{v 1}=73.4 \mathrm{~m} / \mathrm{s}, p_{v 1}=2.28 \mathrm{GPa}, C_{1}=5234 \mathrm{~m} / \mathrm{s} \text {. }
$$

According to formulas (9), the parameters of the SCW are determined when the DW moves parallel to the pipe surface. In the case when the pressure in DP is equal to $p=$ $5 \mathrm{GPa}$, the mass velocity of steel:

$$
u_{v 2}=110 \mathrm{~m} / \mathrm{s}, p_{v 2}=4.52 \mathrm{GPa}, C_{2}=5452 \mathrm{~m} / \mathrm{s} .
$$

If we assume that $p=1 \mathrm{GPa}$, then:

$$
u_{v 3}=24.6 \mathrm{~m} / \mathrm{s}, p_{v 3}=9.78 \cdot 10^{8} \mathrm{~Pa}, C_{3}=5102 \mathrm{~m} / \mathrm{s} .
$$

Thus, the parameters of the SCW in a given pressure range are obtained, which allows us to consider the mechanisms of the processes of destruction of TC8 pieces.

Consider the processes occurring in an explosion in a steel pipe filled with explosives and lumpy waste of tungsten-cobalt alloy TC8.

Let the pipe diameter $d_{1}=100 \mathrm{~mm}$, explosives occupy a part $\beta$ of the pipe volume, and pieces of hard alloy TC 8 are evenly distributed throughout the volume. Obviously, in this case, explosives will detonate in a non-ideal mode. If $\beta=0.3$, the cross-sectional area of the explosive will be $S_{b}=23.56 \mathrm{~cm}^{2}$, which corresponds to a solid charge with a diameter of $54.77 \mathrm{~mm}$. The time for establishing the pressure DP in the pipe, $\bar{p}=\frac{p_{\alpha}}{2}$, where $p_{\alpha}$ is the pressure at the Jouget point, will be within 10-20 $\mu$ s.

When a DW propagates behind its front, a rarefaction wave moves, which is bounded by a gap. The gap moves at a speed approximately equal to $D / 2$. Behind it, the DPs are at rest and their pressure is $0.3 p_{\mathrm{d}}$, where $p_{\mathrm{d}}$ is the pressure at the front of the detonation wave. If we take into account that pieces of TC8 alloy are placed in explosives, it can be 
concluded that they are in a highly inhomogeneous unsteady stress field $\left(10^{10}-10^{12} \mathrm{~Pa} / \mathrm{m}\right.$, $\left.10^{12}-10^{15} \mathrm{~Pa} / \mathrm{s}\right)$.

We will approximate a piece of hard alloy with a ball, and EX - Amonite \#6 GV $\left(p_{\mathrm{d}}=\right.$ $2.5 \mathrm{GPa}, D=4500 \mathrm{~m} / \mathrm{s}$, density of explosive $\rho_{b}=1000 \mathrm{~kg} / \mathrm{m}^{3}$ ). Consider the processes occurring when a DW is falling on a ball. At the point of tangency of the DW of the ball surface, a SCW arises in it, and in the DP a reflected SW. The initial mass velocity of the substance of the ball will be approximately equal to $60 \mathrm{~m} / \mathrm{s}$. Since behind the DW front the pressure decreases according to a power law (for a plane wave, the exponent is 3 , and cylindrical -6 ), this leads to the fact that in a few $\mu$ s the mass velocity decreases several times. In the process of propagation of the DW, the angle of its fall will increase, and the reflection of the SW will become irregular at first, and then Mach. The initial pressure at the front of the SCW will decrease from about $5 \mathrm{GPa}$ to $2.4 \mathrm{GPa}$. The vector of mass velocity during the passage of a wave through the ball will turn to the angle $\pi$. A piece of hard alloy will be destroyed mainly due to cut and shear. The time of destruction is almost independent of the rate of growth of cracks. The minimum size $a$ the particles into which the piece is destroyed can be estimated by the formula:

$$
a \approx \frac{\sigma_{s} h}{p_{\alpha}},
$$

where $h$ is the width of the front of the DW.

In the process of propagation of SCW, the center of mass of the piece will begin to move only after a wave passes through it. The speed of movement is determined by the average mass velocity of the substance behind the wave front. When a compression wave is excited in the piece (there is no flow of matter), the center of mass begins to move after the wave passes through the piece twice.

In the considered processes, it is necessary to estimate the residual pressure DP in the pipe. In pieces TC8 spread SCW. Due to the destruction of matter and internal friction, they are heated by the internal energy of DP, which can be determined by the formula [2]:

$$
U=\frac{p V(1-b)}{n-1}
$$

where $b$-covolume DP, referred to $1 \mathrm{~m}^{3}$. If we take into account that with a steadystate pressure of $25 \mathrm{GPa}$, the TC 8 density increases by $49 \%$, the heating process can be considered isochoric. The decrease in pressure can be determined by the formula:

$$
(1-\beta) \rho V c_{V} \Delta T=\frac{\left(\left(p_{\alpha} / 2\right)-p\right) V \beta(1-\beta)}{n-1},
$$

where $\rho$ is the TC8 density, $c_{V}$ is the specific heat capacity of the alloy, $\Delta T$ is the decrease in the DP temperature, $V$ is the volume of the TC8 pieces. From formula (12) we obtain:

$$
p=\frac{p_{\alpha}}{2}-\frac{(1-\beta)(n-1) \rho c_{V} \Delta T}{\beta(1-b)} .
$$

Covolume $b$ can be determined from the formula [2]:

$$
n=\frac{\gamma}{1-b}
$$

where $\gamma$ is the adiabatic index of DP at atmospheric pressure. 
In [1], it was shown that in SW with pressures at the front from $1 \mathrm{GPa}$ to $5 \mathrm{GPa}$, tungsten carbide is heated from 15 to $199^{\circ} \mathrm{C}$. According to the formula (13) when the alloy is heated at $20-40{ }^{\circ} \mathrm{C}$, the pressure $\mathrm{p}$ will be respectively $0.76-0.26 \mathrm{GPa}$.

When a sufficiently powerful explosive is initiated in a pipe filled with pieces of TC using a detonation cord, then if there is an air gap 4-5 mm thick or $15 \mathrm{~mm}$ sand layer between the concrete fence and the pipe, a discharge wave occurs in the pipe. At the same time, the quality of crushing increases, and the concrete is not destroyed.

Sometimes a pipe with pieces of TC is surrounded by a layer of explosives. Using blasting explosives would cause the pipe to break. Indeed, when the DW reaches the section of the pipe with a piece of TC adjacent to its walls, a SCW arises in the latter with a mass velocity of about $30 \mathrm{~m} / \mathrm{s}$ in it (with $p_{\alpha}=5 \mathrm{GPa}$ ).

In the absence of TC, the pipe walls acquire an initial velocity of about $200 \mathrm{~m} / \mathrm{s}$ (when $\mathrm{SCW}$ is reflected from the wall, the velocity increases by about two times), which leads to the wall breaking.

If dynamones are used as explosives (blasting agents), then the average mass velocity of steel is several $\mathrm{m} / \mathrm{s}$. The pipe is crimped, and pieces of TC are destroyed, mainly from quasi-static loads. At the same time, the quality of crushing and softening is low. Especially low quality crushing and softening is observed in the case of using an open charge. Calculations show that in this case only a quarter of explosives (the active part of the charge) is used for destruction.

\section{Conclusions}

For explosive crushing and softening of pieces of solid TC alloys, it is necessary to create fields in them with stress of $1-10 \mathrm{GPa}$, inhomogeneities of $10^{10}-10^{12} \mathrm{~Pa} / \mathrm{m}$, and a rate of change of stress of $10^{10}-10^{15} \mathrm{~Pa} / \mathrm{s}$. The most effective crushing of pieces of hard alloys in the case when the explosive is inside them.

If the alloys contain $\mathrm{Ni}, \mathrm{Mo}$, and other metals, the $A, m$ coefficients in the shock compressibility equation can be determined using table 13 in [1]. The velocity of the stress waves in such alloys is found by the formula (3), which in this case can be written for several components.

The work obtained the results needed by experimenters and explosives practitioners for developing designs of crushing charges for pieces of tungsten-cobalt alloys.

\section{References}

1. Stanyukovich, K.P., Baum, F.A., Shekhter, B. I. (2013). Phizika vzryva. Ripol Classic

2. Kurinnoy, V.P., Garkusha, I.P. (2012). Adiabatic equation for detonation products of explosives and explosion products with additives. Geo-Technical Mechanics, 104, 112 119 